\title{
Crianças operadas e condições familiares. O que muda? Estudo no Instituto do Coração-HCFMUSP
}

\author{
Lais S. CROCHIK ${ }^{\star}$, Iris F. BERTANI*, Miguel BARBERO-MARCIAL*
}

RBCCV $44205-279$

CROCHIK, L. S.; BERTANI, I. F.; BARBERO-MARCIAL, M. - Crianças operadas e condiçōes famíliares. O que muda? Estudo no Instituto do Coração-HCFMUSP. Rev. Bras. Cir. Cardiovasc., 10 (4): 206$210,1995$.

RESUMO: No período de março a agosto de 1993, 57 familiares de crianças com idades entre 0 e 7 anos portadoras de cardiopatias congênitas, internadas para tratamento cirúrgico, foram entrevistados. Destes, $94,5 \%$ procedem da zona urbana e em $86 \%$ o grupo familiar não ultrapassa 5 pessoas. Das famílias estudadas, $56,5 \%$ dos pais e $58 \%$ das mães apresentam instrução primária e $41 \%$ dos pais possuem ocupação de nível técnico e $72 \%$ das mães não têm qualificação profissional. Foram estudados os seguintes ítens: a) mudanças profissionais dos pais após o nascimento do filho; $b$ ) alterações no relacionamento interpessoal; c) dificuldades para enfrentar o momento da cirurgia; d) expectativas futuras com o filho operado. Os resultados mostraram que, para os casais jovens de classe social média baixa e em fase de estruturação familiar, o aparecimento de um membro doente e o evento da cirurgia cardíaca identificam áreas de estresse e alguns evitam falar do futuro.

DESCRITORES: Familia e crianças operadas. Familia e crianças hospitalizadas. Comportamento familiar e crianças operadas.

\section{INTRODUÇÃO}

As condições demográficas e sócioeconômicas da população dos países em desenvolvimento estão associadas a padrões de vida insatisfatórios e a barreiras na solução da questão da doença nas famílias ${ }^{8}$.

Outros estudos, como os de FREITAS ${ }^{1}$ dedicam-se à mesma análise das deficiências da estrutura de atendimento à saúde da criança no Brasil, relacionando-as a problemas sócioeconômicos.

Muito pouco se escreveu, contudo, a respeito de crianças internadas na expectativa de uma operação, notadamente crianças cardíacas e suas familias.
Levantamentos bibliográficos nas bases de dados internacionais (MEDLINE, LILACS) e nacionais muito pouco acrescentaram às indagações iniciais.

Obteve-se, em SAUNDERS ${ }^{10}$, a descrição do trabalho das Assistentes Sociais com crianças com diversos graus de incapacidade e suas famílias.

Nesta perspectiva há o trabalho de MONTEIRO et alii ${ }^{5}$ realizado com mães acompanhantes de crianças internadas.

Foram, também, objeto de análise as repercussões psicológicas da hospitalização da criança e sua família ${ }^{6}$. Já a preocupação de não realizar um trabalho visando apenas psicologizar o atendimento

\footnotetext{
Trabalho realizado no Instituto do Coração do Hospital das Clinicas da Faculdade de Medicina da Universidade de São Paulo. São Paulo, SP, Brasil. * Do Instituto do Coração do Hospital das Clínicas da Faculdade de Medicina da Universidade de São Paulo. Recebido para publicação em agosto de 1995.

Endereço para correspondência: Laís S. Crochik. Serviço Social Médico. Av. Dr. Enéas de Carvalho Aguiar, 44 - Cerqueira Cesar - 05403-000 - São Paulo - SP - Brasil
} 
CROCHIK, L. S.; BERTANI, I. F.; BARBERO-MARCIAL, M. - Crianças operadas e condiçōes famíliares. O que muda? Estudo no Instituto do Coração-HCFMUSP. Rev. Bras. Cir. Cardiovasc., 10 (4):206-210, 1995.

foi apresentado nos estudos de REDOGLIA et alii ${ }^{7}$ e SANTOS et alii ${ }^{9}$, permitindo uma abordagem sobre a relação da equipe multiprofissional, família e tratamento, na questão da hospitalização da criança.

Em outro estudo relacionado à hospitalização de crianças e mais próximo ao objetivo da atual pesquisa, observou-se os trabalhos de SUAREZ \& ARRIAGA ${ }^{11}$ sobre o nivel de informação dos pais em relação a doença do filho.

Foi, contudo, nos manuais elaborados para orientação de pais de crianças hospitalizadas que se descreve sobre a cirurgia e o seu impacto sobre a vida familiar, principalmente enfocando-se sentimentos e emoções dos pais frente à situação ${ }^{3}$, bem como o papel da avó como elemento de apoio da nova família?

Esta pesquisa, contudo, para responder aos objetivos a que se propõe, embasou-se mais particularmente na experiência prática desenvolvida pela Assistente Social da Unidade de Internação Infantil com pais de crianças cardíacas operadas e a reviravolta que esta condição provocou em suas vidas.

O comportamento dos pais diante do aparecimento, na família, de uma criança com doença cardíaca e a necessidade de uma cirurgia de peito aberto, os cuidados que essa criança necessita, são fatores que, diante do quadro social do País, provocam mudanças em toda a estrutura familiar. É este o aspecto que foi verificado no presente estudo.

\section{CASUÍSTICA E MÉTODOS}

O estudo foi realizado nas Unidades Infantis do Instituto do Coração do HC/FMUSP com todas as crianças internadas, com idade variando entre 1 dia e 7 anos e operadas no período compreendido entre fevereiro e agosto de 1993. Foram estudadas 57 famílias.

O número de crianças foi determinado por estudo baseado no global de-crianças internadas por ano nas Unidades Infantis e, submetidas a cirurgia cardíaca.

Após autorização e concordância dos pais ou responsáveis, era preenchido questionário composto de 11 questões abordando a posição do paciente na composição familiar, a procedência da família, o grau de conhecimento dos pais sobre cardiopatia de seu filho, nível de intercomunicação entre os membros da família sobre o problema e as mudanças ocorridas após o nascimento da criança, e as dificuldades e facilidades durante o período de hospitalização e cirurgia.

\section{RESULTADOS}

Das 57 famílias estudadas, $30(53 \%)$ crianças eram do sexo masculino e 27 (48\%) do sexo feminino.

As crianças provêm de famílias com vinculação ao sistema público de saúde em $55(97 \%$ ) casos e as demais na categoria de atendimento particular ou de convênio de assistência médica. Quanto ao número de pessoas das famílias, são consideradas em $49(86 \%)$ como de médio porte, com até 5 pessoas e em $8(14 \%)$ de grande porte com até 9 pessoas. Estas famílias são estruturadas em 53 (93\%) com a presença de pai e mãe e em $4(7 \%)$ apenas com a mãe.

A idade dos pais variou de 18 anos a 54 anos, sendo a faixa predominante encontrada entre 26 anos e $35(49 \%)$ anos. As mães apresentam idades entre 19 anos e 43 anos, com igual predominância entre 26 anos e $35(55 \%)$ anos.

Com relação à escolaridade, temos uma concentração de $25(47 \%)$ pais e $29(51 \%)$ mães com instrução até $1^{\circ}$ grau incompleto.

Quanto à ocupação, verificou-se uma concentração de $22(41 \%)$ pais no nível técnico, enquanto que as mães apresentam-se preferencialmente sem qualificação profissional em $41(72 \%)$; entre esses casos, 38 mães são prendas domésticas.

TABELA 1

MUDANÇAS OCORRIDAS NA FAMÍLIA E COMPOSIÇĀO FAMILIAR

\begin{tabular}{lrrr}
\hline \multicolumn{1}{l}{ Mudança } & & No de Membros & \\
\hline & 2 a 5 & 6 a 9 & TOTAL \\
Vida profissional & $2(4,0 \%)$ & $1(12,5 \%)$ & $(5,0 \%)$ \\
Plano de vida & $27(55,0 \%)$ & $3(37,5 \%)$ & $30(53,0 \%)$ \\
Financeiro & $5(10,0 \%)$ & $5(10,0 \%)$ & $11(19,0 \%)$ \\
Relacionamento & $9(18,5 \%)$ & $2(25,0 \%)$ & $8(14,0 \%)$ \\
Nada mudou & $6(12,0 \%)$ & $2(25,0 \%)$ & $\mathbf{5 7 ( 1 0 0 , 0 \% )}$ \\
\hline Total & $\mathbf{4 9 ( 8 6 , 0 \% )}$ & $\mathbf{8 ( 1 4 , 0 \% )}$ & \\
\hline
\end{tabular}


CROCHIK, L. S.; BERTANI, I. F.; BARBERO-MARCIAL, M. - Crianças operadas e condiçōes familiares. O que muda? Estudo no Instituto do Coração-HCFMUSP. Rev. Bras. Cir. Cardiovasc., 10 (4): 206-210, 1995.

TABELA 2

MUDANÇAS OCORRIDAS NA FAMILIA E DIAGNÓSTICO DA DOENÇA DA CRIANÇA

\begin{tabular}{|c|c|c|c|c|c|}
\hline Mudança & & & agnóstico & & \\
\hline & CIANOG. & ACIANOG. & ADQUIR. & ARRIT. & TOTAL \\
\hline Vida profissional & $2(5,5 \%)$ & $1(6,0 \%)$ & - & - & $3(5,0 \%)$ \\
\hline Plano de vida & $18(49,0 \%)$ & $10(62,5 \%)$ & $1(3,5 \%)$ & $1(100,0 \%)$ & $30(52,5 \%)$ \\
\hline Financeiro & $2(5,5 \%)$ & $2(12,5 \%)$ & $1(33,0 \%)$ & & $5(9,0 \%)$ \\
\hline Relacionamento & $10(27,0 \%)$ & $1(6,0 \%)$ & - & - & $11(19,0 \%)$ \\
\hline Nada mudou & $5(13,5 \%)$ & $2(12,5 \%)$ & $1(33,5 \%)$ & - & $8(14,0 \%)$ \\
\hline Total & $37(65,0 \%)$ & $16(28,0 \%)$ & $3(3,5 \%)$ & $1(2,0 \%)$ & $57(100,0 \%)$ \\
\hline
\end{tabular}

O estudo abordou crianças portadoras de cardiopatias congênitas e adquiridas, diagnosticadas com idade de 1 mês a $11(47 \%)$ meses e em $5(8 \%)$ casos com mais de 12 meses.

Quanto ao nível de informação sobre a doença do filho, temos que tanto os pais como as mães sabem que se trata de problema de formação genética. Como padrão de intercomunicação familiar, observa-se que 29 (51\%) dos entrevistados conversam com o cônjuge sobre a doença do filho, seguido em $7(12 \%)$ por conversa com todos os familiares e em $6(10 \%)$ com os avós.

Das emoções expressadas pelos pais, $24(42 \%)$ referem que a situação hospitalar mais difícil de enfrentar durante o período de internação foi o momento da cirurgia, seguido por 6 respostas referentes ao tratamento em si e tudo o que ele envolve.

Em contrapartida, foi o pós-operatório que proporcionou aos pais, em $39(68 \%)$, maior tranqüilidade durante a internação hospitalar dos filhos doentes.

Aconteceram mudanças na vida destas famílias, com a doença de uma de suas crianças, que foram observadas na vida profissional dos pais em $3(5 \%)$ nos planos de vida em $30(52 \%)$, na situação financeira em $5(9 \%)$, no relacionamento interpessoal em $11(19 \%)$, enquanto que, em 8 (14\%), nada mudou.
Como se observa na Tabela 1, o aspecto familiar mais afetado pela doença da criança foi o relacionado aos planos de vida da família. O segundo aspecto predominante foi alteração nos padröes de relacionamento familiar.

Nota-se indice significativo de respostas "nada mudou".

Observamos, na Tabela 2, que, mesmo diante de diagnósticos diferenciados, são os planos de vida familiar que se alteram.

Não foram observadas na Tabela 3 , outras alterações diferentes nas mudanças ocorridas na família conforme o grau de instrução dos pais.

Em todos os níveis educacionais, a maior concentração de respostas sobre mudanças se deu na variável "plano de vida". A resposta "relacionamento" apresentou maior concentração, para os pais com $1^{\circ} \mathrm{grau}$ incompleto. Da mesma forma, as respostas "nada mudou" foram encontradas, preferencialmente, entre pais do mesmo nivel de escolaridade $\left(1^{\circ}\right.$ grau).

As mudanças de planos de vida (Tabela 4) estão concentradas em pais com ocupações de nível técnico e aqueles sem qualificação profissional. No mesmo nível ocupacional concentram-se as respostas relativas ao relacionamento.

TABELA 3

MUDANÇAS OCORRIDAS NA FAMÍLIA E GRAU DE INSTRUÇĀO DOS PAIS

\begin{tabular}{|c|c|c|c|c|c|}
\hline \multirow[t]{2}{*}{ Mudança } & \multicolumn{5}{|c|}{ Grau de Instrução } \\
\hline & $\begin{array}{r}\text { SEMI } \\
\text { ALFAB. }\end{array}$ & $1^{\circ} \mathrm{GRAU}$ & $2^{\circ} \mathrm{GRAU}$ & SUPERIOR & TOTAL \\
\hline Vida profissional & - & $6(100 \%)$ & - & - & $6(5,5 \%)$ \\
\hline Plano de vida & $4(7,0 \%)$ & $23(40,0 \%)$ & $21(37,0 \%)$ & $9(16,0 \%)$ & $57(52,0 \%)$ \\
\hline Financeiro & - & $5(55,5 \%)$ & $4(44,5 \%)$ & - & $9(8,0 \%)$ \\
\hline Relacionamento & - & $14(63,5 \%)$ & $6(27,0 \%)$ & $2(9,0 \%)$ & $22(20,0 \%)$ \\
\hline Nada mudou & - & $15(94,0 \%)$ & $1(2,0 \%)$ & - & $16(14,5 \%)$ \\
\hline Total & $4(3,5 \%)$ & $63(57,0 \%)$ & $32(29,0 \%)$ & $11(10,0 \%)$ & $110(100,0 \%)$ \\
\hline
\end{tabular}


CROCHIK, L. S.; BERTANI, I. F.; BARBERO-MARCIAL, M. - Crianças operadas e condiçōes famíliares. O que muda? Estudo no Instituto do Coração-HCFMUSP. Rev. Bras. Cir. Cardiovasc., 10 (4):206-210, 1995.

TABELA 4

MUDANÇAS OCORRIDAS NA FAMÍLIA E OCUPAÇÃO DOS PAIS

\begin{tabular}{|c|c|c|c|c|c|}
\hline Mudança & & $t 8$ & Ocupação & & \\
\hline & $\begin{array}{l}\text { NÍVEL } \\
\text { SUPERIOR }\end{array}$ & $\begin{array}{c}\text { SEMI- } \\
\text { QUALIFIC. }\end{array}$ & TÉCNICO & $\begin{array}{l}\text { DESEMPRE- } \\
\text { GADOS }\end{array}$ & TOTAL \\
\hline Vida profissional & - & $5(8,0 \%)$ & $1(4,0 \%)$ & - & $6(5,5 \%)$ \\
\hline Plano de Vida & $8(66,5 \%)$ & $30(47,5 \%)$ & $14(52,0 \%)$ & $5(62,5 \%)$ & $57(52,0 \%)$ \\
\hline Financeiro & $1(8,5 \%)$ & $4(6,5 \%)$ & $3(11,0 \%)$ & $1(12,5 \%)$ & $9(8,0 \%)$ \\
\hline Relacionamento & $3(25,0 \%)$ & $13(20,5 \%)$ & $4(15,0 \%)$ & $2(25,0 \%)$ & $22(20,0 \%)$ \\
\hline Nada mudou & - & $11(17,5 \%)$ & $5(18,5 \%)$ & - & $16(14,5 \%)$ \\
\hline Total & $12(12,0 \%)$ & $63(57,0 \%)$ & $27(24,5 \%)$ & $8(7,0 \%)$ & $110(100,0 \%)$ \\
\hline
\end{tabular}

\section{COMENTÁRIOS}

Diante das dificuldades socioeconômicas decorrentes de deficiente estrutura e padrōes insatisfatórios brasileiros de assistência pública à saúde, pais de crianças cardíacas operadas sentem-se em alguns momentos desamparados, precisam rever planos de vida, reformular projetos pessoais e desistir de sonhos profissionais.

Estes pais vivem, na maioria das vezes, em condições de estresse e dificuldades. São, quase sempre, jovens e em fase de construção de seus projetos de vida.

A existência de uma criança na família com necessidade de ser submetida a um procedimento cirúrgico tão invasivo modifica o padrão de vida da nova família, altera seus planos e fortalece (agudiza) padrōes comportamentais que permaneciam latentes no relacionamento cotidiano.

É a estes pais que se exige, repentinamente, que apresentem equilíbrio e apoio mútuo, além de prestarem cuidados ao filho doente, sem descuida- rem de outros filhos. A família passa por um teste de sobrevivência; algumas crianças, porém, já vêm de lares onde a mãe é o único membro a apoiar o filho doente. O sofrimento decorrente da notícia da existência da cardiopatia do filho e a necessidade de cirurgia podem levar a família a reformular seus planos de vida. Alguns pais referem que nada mudou, como se nada tivesse acontecido em suas vidas. Esta resposta parece demonstrar muito mais a falta de reflexão dos pais ou negação sobre a nova situação na vida da família. São estes os pais que apresentam menor qualificação profissional $e, a o$ mesmo tempo, que mais mudam de ocupação, numa evidente contradição de sua afirmação inicial.

É visando a uma clarificação e integração desta família junto ao momento que está enfrentando na hospitalização, que a Equipe Multiprofissional que atua na Unidade Infantil intervém, através de reuniōes semanais com estes pais. $\mathrm{Na}$ ocasião, são refletidos pontos abordados nesta pesquisa, como também outros aspectos que são trabalhados pela Equipe a nível grupal ou individualmente. 
CROCHIK, L. S.; BERTANI, I. F.; BARBERO-MARCIAL, M. - Crianças operadas e condiçōes famíliares. O que muda? Estudo no Instituto do Coração-HCFMUSP. Rev. Bras. Cir. Cardiovasc., 10 (4): 206-210, 1995.

\section{RBCCV 44205-279}

CROCHIK, L. S.; BERTANI, I. F.; BARBERO-MARCIAL, M. - Operated children and familiar conditions. What changes? A study in the Heart Institute. University of São Paulo, Brazil. Rev. Bras. Cir. Cardiovasc., $10(4): 206-210,1995$.

ABSTRACT: From March to August 1993, parents of 57 children between 0 and 7 years old, with congenital cardiopathies, hospitalized for surgical treatment were studied; $94.5 \%$ came from the urban zone and $86 \%$ from familiar groups who not exceeded 5 people. In the familiar group, $56.5 \%$ of the fathers and $58 \%$ of the mothers had elementary school; $41 \%$ of the fathers had technical occupation and $72 \%$ of the mothers had no professional qualification (housewives). The following subjects were studied: a) professional changes of parents; b) modifying in interpersonal relationship; c) difficulties fo face the surgery moment; d) future hopes related to the surgical child. The results showed that, for young couples of low medium social class, concerning the stage of familiar structuring, a sick member and the fact of the cardiac surgery, present stress and some avoid to speak about the future.

DESCRIPTORS: Family and surgical treated children. Family and hospitalized children.

\section{REFERÊNCIAS BIBLIOGRÁFICAS}

1 FREITAS, C. B. D. - Estrutura do atendimento à saúde da criança no Brasil. Rev. Bras. de Crescimento e Desenvolvimento Humano, 3(1): 64-76, 1993.

2 HEART CHILDREN: a practical handbook for parents of children with congenital heart problems. Camberley, Heart Line Association, 1989.

3 KLAUS, M. H. - La relacion madre-hijo. Buenos Aires, Editorial Médica Panamericana, 1978.

4 LAMOSA, B. W. R. - Abordagem psicológica da criança cardiopata. Cardiologia Pediátrica. São Paulo, 1984. p. 279-281.

5 MONTEIRO Fo, L.; LOPES NETO, A. A; RANGEL, A. M. H; MONTEIRO, M. T. S. - O programa de hospitalização da criança acompanhada (PHOCA) do Hospital Municipal Souza Aguiar. J. Pediatria, 64: 242-247, 1988.

6 MOUTH, R \& SILVA, S. L. A. - Repercussões psicológicas da hospitalização na criança e sua família. Pediatria Moderna, 19(8), 1984.
7 REDOGLIA, S.; RANNA, N.; TULHA, O. M. P. A. T.; NOSEK, L. - Relato de uma experiência com grupo de pais de crianças internadas com patologia clínica. Rev. Pediatria, 1: 61-68, 1979.

8 RODRIGUES F?, J.; COSTA, W.; IENO, G. M. L. Determinantes de utilização do cuidado pré-natal entre famílias de baixa renda no Estado do Paraná, Brasil. Rev. Saúde Pública, 28: 284-289, 1994.

9 SANTOS, M. E. R. - A hospitalização da criança: a visāo do familiar. J. Pediatria, 56: 391-395, 1984.

10 SAUNDERS, E. J. - Services for infants and toddlers with disabilities: idea. Part. H. National Association of Social Workers, 20 (1): 39-45, 1995.

11. SUAREZ, R. S. R. \& ARRIAGA, A. L. - Cuanto saben los padres sobre la enfermedad de su hijo hospitalizado? Bol. Méd. Hospital Infantil México, 42: 226-233, 1985.

12 ZIOLKO, M. E. - Counseling parents of children with disabilities: a review of the literature and implications for practice. J. Reabilitation, 57: 29-34, 1991. 